\title{
Development of an Integrated Data Acquisition System for a Small Flight Probe
}

\author{
Gregory T. Swanson ${ }^{1}$, Daniel M. Empey ${ }^{2}$, Kristina A. Skokova ${ }^{3}$, Ethiraj Venkatapathy ${ }^{4}$ \\ NASA Ames Research Center, Moffett Field, CA, 94035, USA
}

\begin{abstract}
In support of the small probe re-entry investigation for thermal protection system engineering (SPRITE) concept, an integrated data acquisition system has been developed and fabricated for preliminary testing. The data acquisition system has been designed to condition traditional thermal protection system sensors, store their data to an on-board memory card, and in parallel, telemeter to an external system. This first step in developing a flight-ready, cost-effective data acquisition system is for small probe flight testing. In the fall of 2010, this system was integrated into a $14 \mathrm{in}$. diameter, 45 degree sphere cone probe instrumented with thermal protection system sensors. This system was then tested at the NASA Ames Research Center Aerodynamic Heating Facility's arc jet at approximately 170 $\mathrm{W} / \mathrm{cm}^{2}$. The first test in December 2010 highlighted hardware design flaws that were later redesigned and implemented leading to a successful test in February 2011.
\end{abstract}

\section{Nomenclature}

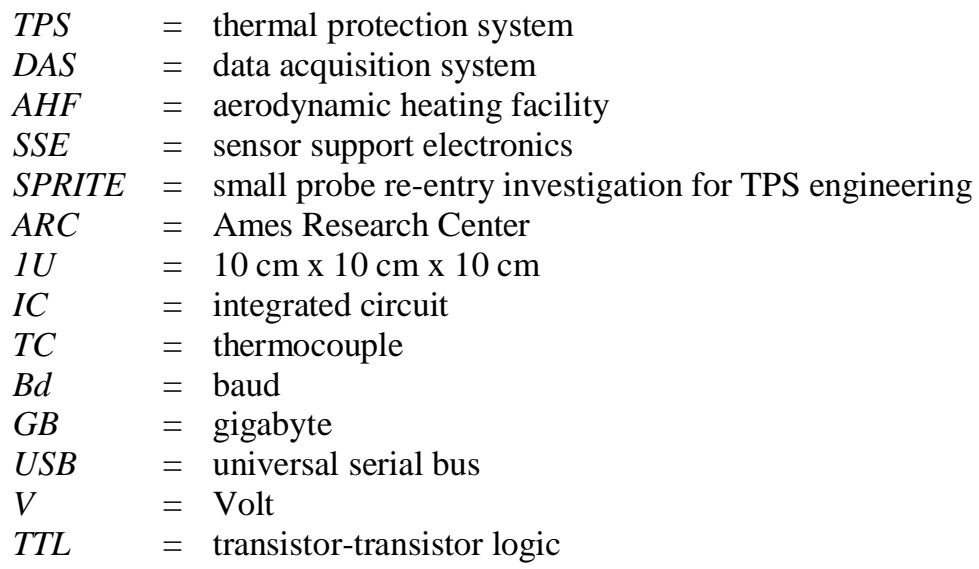

\section{Introduction}

\footnotetext{
$\mathrm{C}$ URRENT space exploration is pressing the boundaries of proven flight capabilities resulting in a push to develop new and enhanced technologies that will enable greater opportunities in future missions. One key technology that requires innovation is a spacecraft's thermal protection system (TPS). Development of new TPS materials requires a significant verification effort. Traditional TPS material validation has been completed mainly through coupon ground tests, and when possible, at a much greater cost, full-scale flight tests. Full-scale flight tests provide the most relevant characterization, however with ever-increasing federal budgetary constraints a greater emphasis is being placed on the more economic test programs. When employed, ground testing in arc jet facilities provides a relatively inexpensive method for TPS material characterization. Unfortunately the arcjet environments produced only partially simulate atmospheric entry. In addition, ground testing traditionally utilizes TPS material coupons, lacking geometric parameters that would be prevalent in a full-scale model. Reference [1] discusses the

${ }^{1}$ Electrical Engineer, Entry Systems and Vehicle Development Branch, ERC Inc., M/S 230-3, Student Member

${ }^{2}$ Senior Engineer, Thermo-Physics Facilities Branch, M/S 229-4, Jacobs Technology, M/S 229-4

${ }^{3}$ Materials Research Scientist, Thermal Protection Materials Branch, ERC Inc., M/S 234-1

${ }^{4}$ Chief Technologist, Entry Systems and Technologies Division, NASA, MS 229-3, Associate Fellow AIAA
} 
SPRITE (Small Probe Re-entry Investigation for TPS Engineering) concept in detail. To better correlate ground testing to full-scale flight testing, a small probe TPS test bed project has been initiated.

SPRITE [2] is scaled to allow testing of the same exact sized probe in ground and flight testing on a relatively inexpensive platform. This will provide essential TPS validation and a better understanding of the differences present between ground and flight testing. To advance the SPRITE concept, a probe was designed, fabricated and tested in the Aerodynamic Heating Facility (AHF) at NASA Ames Research Center (ARC). The probe featured instrumentation plugs to characterize the TPS's performance during the ground test effort. To collect the TPS sensor data in real-time, a custom integrated data acquisition system (DAS) was developed in parallel to the SPRITE probe. SPRITE's small geometry, unique performance, and AHF-related design requirements precluded the use of COTS hardware for most DAS components. This paper details the design and testing of a custom integrated DAS for small flight probes.

\section{Data Acquisition System Design Requirements}

There were five primary requirements. Requirements 1, 2 and 3 were specified to support the SPRITE proof-ofconcept ground test:

1) The DAS shall support and condition traditional TPS sensors, such as thermocouples

2) The DAS shall telemeter the real-time data via a serial connection

3) The DAS shall not exceed a $1 \mathrm{U}$ unit cubesat volume

Additional requirements were included to address the future challenges of a SPRITE flight test. Requirements 4 and 5 were chosen because they could be demonstrated within the modest budget of this ground test:

4) The DAS shall store the real-time sensor data to on-board memory

5) The DAS shall be battery powered

\section{Data Acquisition System Hardware Design}

To develop the small probe integrated DAS hardware, the effort was divided into three parts; the sensor support electronics and data handling board (SSE\&DH), the power interface and distribution board, and the high-capacity memory board.

\section{A. Sensor Support Electronics and Data Handling Board}

The SSE\&DH is a custom designed, $90 \mathrm{~mm}$ by $90 \mathrm{~mm}$, four layer printed circuit board (PCB), and acts as the heart and soul of the small probe DAS. The SSE\&DH interfaces and conditions up to 40 sensors whose data sets are then organized by a PIC18F4520 microprocessor. The microprocessor writes the real-time sensor data to the highcapacity memory board and in parallel telemeters via a serial connection.

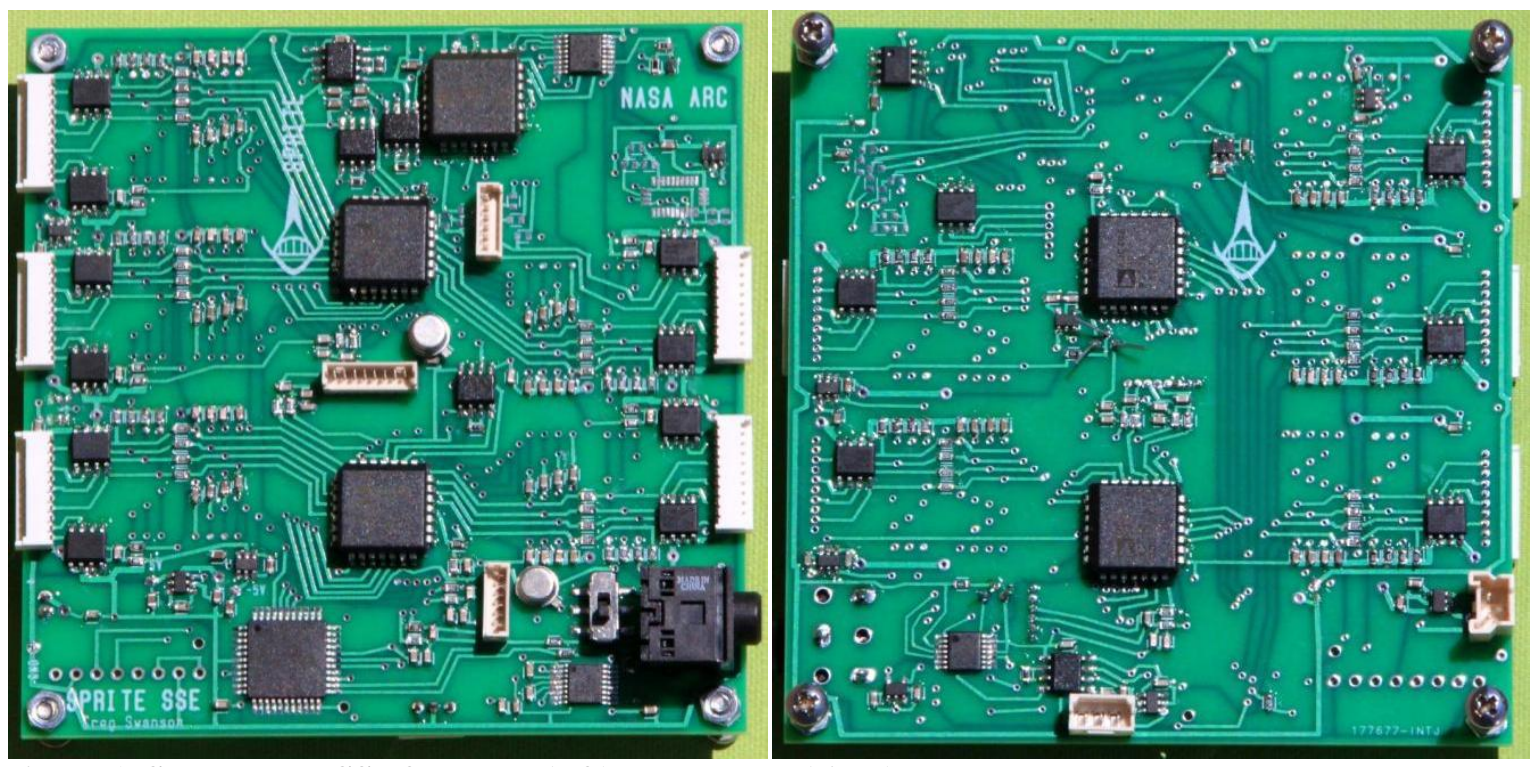

Figure 1. Small probe SSE\&DH top (left) and bottom (right) layout. 
The SSE\&DH sensor capability includes thermocouples (TCs), isotherm sensors, integrated circuit (IC) temperature sensors, a triaxial accelerometer, and a triaxial gyro. The board has been designed to incorporate 10 housekeeping TCs. These TCs are reserved to make housekeeping temperature measurements inside the probe such as battery and component temperatures. Each housekeeping TC is low pass filtered. Additionally the SSE\&DH provides 20 TPS TC connections. These 20 TCs are low pass filtered as well, but also include transient voltage protection and open circuit detection. The transient voltage protection protects the SSE\&DH against any electrical phenomena that would cause large transient voltage spikes that could otherwise harm or destroy the circuit components. Open circuit detection is provided to indicate TC burn through. In ablative TPS materials, depending on the environment and depth of the TC, there is a possibility that the TC will become exposed to the flow field burning through its welded junction. Knowing this point in time is beneficial in characterizing the TPS material performance. The DAS can manage up to 5 TPS Isotherm sensors [6]. These sensors can provide insight into the ablative performance of TPS materials and are similar in conditioning circuit design as the TPS TCs. They require transient voltage protection, low pass filtering, and a high accuracy current source to drive the sensors. Additionally three IC temperature sensors have been employed on the SSE\&DH to provide cold junction correction for the 30 thermocouples.

All sensor data is amplified and multiplexed into the PIC microprocessor where it is organized and distributed at $9600 \mathrm{Bd}$ to the high-capacity memory board. In parallel, the SSE\&DH also sends the real-time sensor data to an external source using a RS-232 connection.

\section{B. Power Interface Board}

The custom designed power interface board is a $40 \mathrm{~mm}$ by $28 \mathrm{~mm}$ two-layer PCB. It conditions the battery power and then distributes it for the entire small probe DAS. The board interfaces with two $3.7 \mathrm{~V}$ lithium polymer batteries. Their supplied power is then run in series to provide a raw $7.4 \mathrm{~V}$. The power interface board then conditions the raw battery voltage into positive $5.0 \mathrm{~V}$, negative $5.0 \mathrm{~V}$, and positive $3.3 \mathrm{~V}$ that are required by the DAS components. The board includes a switch for easy power on/off function.

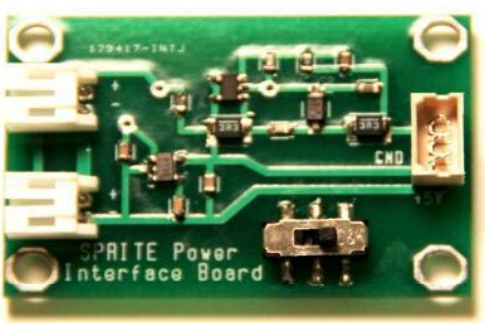

Figure 2. Power interface board.

\section{High-Capacity Memory Board}

To meet the DAS goals a commercial off the shelf high-capacity memory board was procured and modified to interface with the SSE\&DH. The memory board is $60 \mathrm{~mm}$ by $38 \mathrm{~mm}$. It is capable of data storage up to $2 \mathrm{~GB}$ using

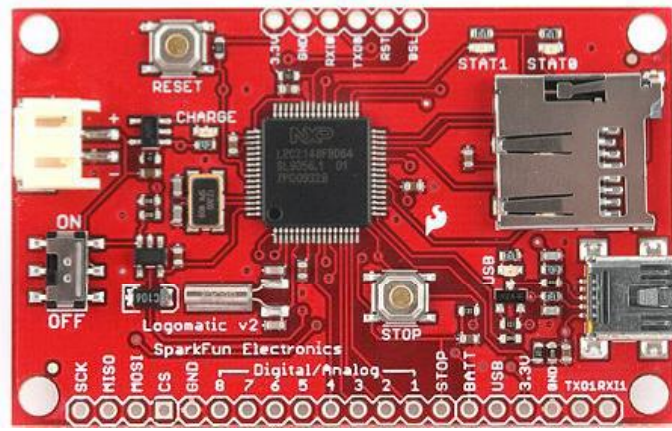

Figure 3. High-capacity memory board. a non-volatile memory card. The memory board employs an ARM 7 microprocessor with two data buffers. The PIC micro-processor on the SSE\&DH sends the organized sensor data to one buffer until the 500 byte limit is reached, at which point the data in the full buffer is written to the memory card, while in parallel the second data buffer begins receiving data. Once it becomes full the ARM 7 again writes the data to memory while in parallel the first data buffer again commences receiving data. This process continues back and forth until the DAS is powered off. The data can then be retrieved from the high-capacity memory board via a micro USB connection, or by removing the memory card and using a separate reading device.

\section{Data Acquisition System Testing and Results}

In 2010, two 14 in. diameter, 45 degree half-angle sphere cone probes were designed and fabricated for testing in the NASA ARC AHF's arc jet under the SPRITE program. Both probes were designed with an aluminum carrier structure, an ablative forebody TPS, and an insulative backshell TPS. Each probe was equipped with three TPS instrumentation plugs located at the stagnation point, mid-cone, and bottom-cone as depicted in Figure 4. The stagnation TPS plug was instrumented with three TCs at varied depths and one isotherm sensor. The mid- and bottom-cone plugs were also instrumented with three TCs at varied depths. An additional eight TCs were welded to the back plane of the carrier structure to provide further insight into the SPRITE probe's performance. This is 


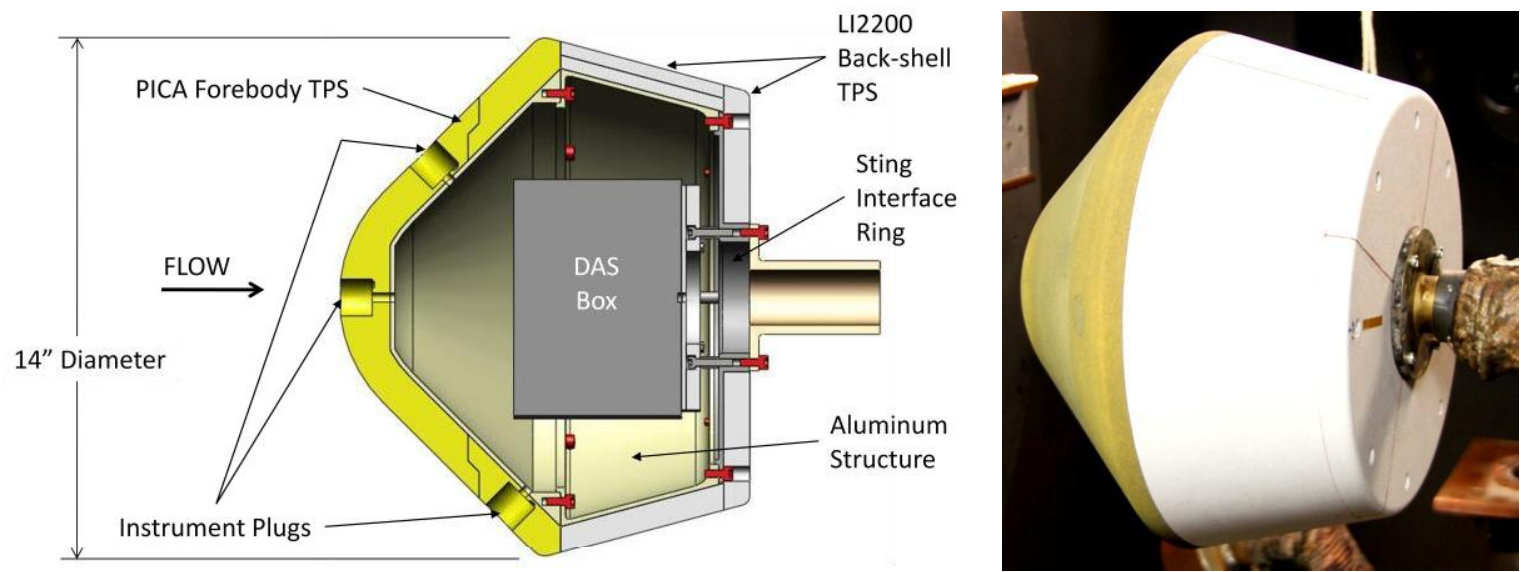

Figure 4. Cross-section of the SPRITE small probe design (left) and fabricated model (right).

detailed in Figure 5. Inside the probe an aluminum box was designed to encase the small probe DAS. The box was thermally isolated from the carrier structure using a Teflon disk to mitigate DAS and battery overheating. The DAS box included three bonded TCs to provide feedback on the thermal model [4]. On the backside of the probe a ring interface was designed to mate with the sting arm that would hold the assembly during arc jet testing.

\section{A. SPRITE Test 1 Setup}

The first SPRITE probe was tested at the NASA ARC AHF's arc jet in December 2010. The test characterized the small probe's performance in an aerothermal heating environment of approximately $170 \mathrm{~W} / \mathrm{cm}^{2}$ for 50 seconds. To evaluate the SPRITE systems' performance, 25 TCs and one isotherm sensor were monitored real-time by both the small probe DAS and the AHF data system. 12 TCs and the isotherm sensor were conditioned and recorded by the small probe DAS, while the other 13 TCs were monitored by the AHF data system. A diagram of the sensor placement and ownership is depicted in Figure 5. A further detailed description of the sensor layout can be found in reference [3].
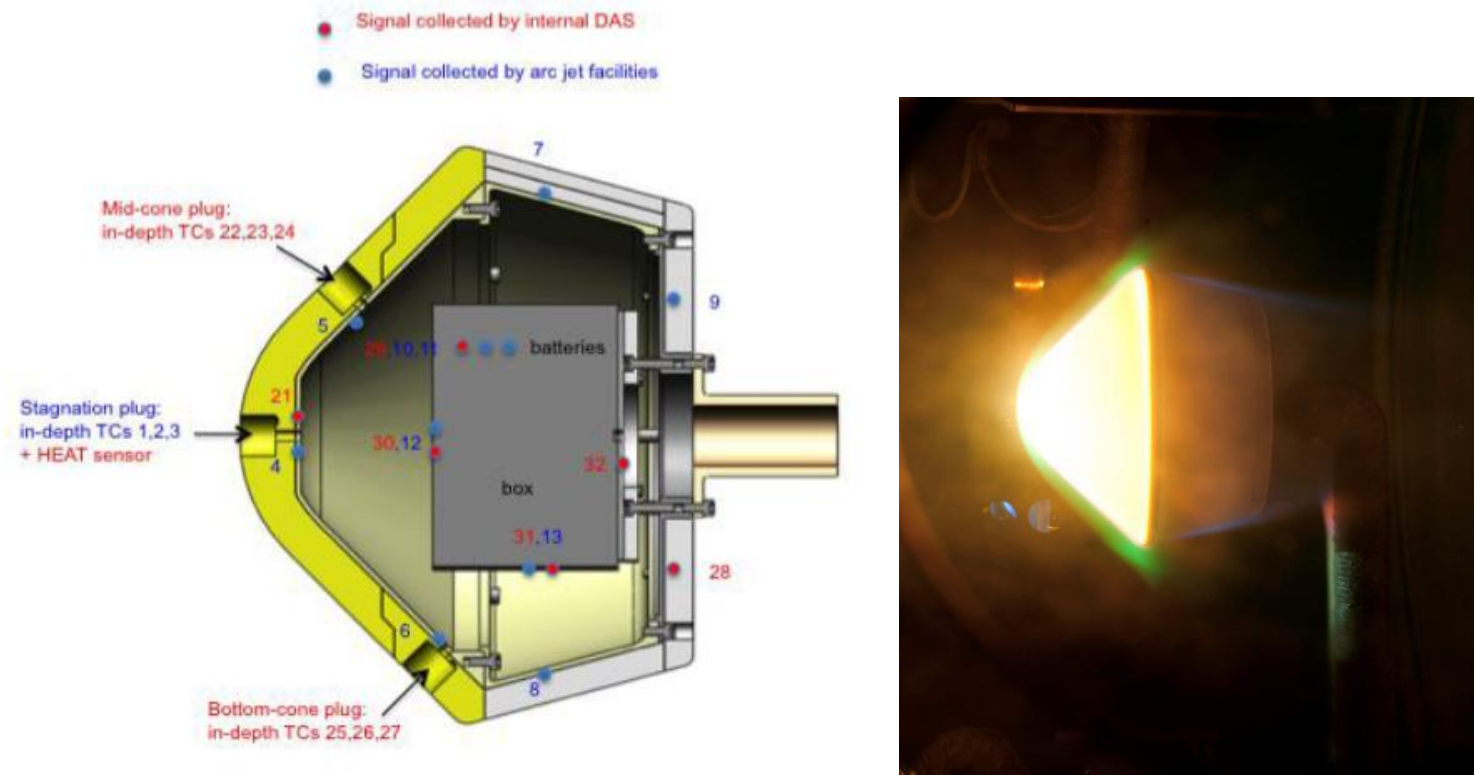

Figure 5. Sensor layout for SPRITE test 1 (left) and a picture of the arc jet testing of probe (right).

As described previously, the custom DAS was located inside the small probe to condition and store sensor data to the high-capacity memory board, and in parallel, telemeter the same data utilizing a RS-232 protocol. Normally this serial connection is run directly to a laptop base station via a USB cable, but for safety purposes the AHF regulations do not allow electrically conductive paths to run from the inside to the outside of the arc jet chamber. To circumvent this constraint, two RS-232 to fiber optic converters were employed to satisfy the safety requirement by feeding data from inside to the outside of the arc jet chamber through optical fiber cables.

To enhance AHF test operations, two additional DAS hardware features were integrated. To mitigate battery power loss during the lengthy installation, an external switch was implemented allowing the SPRITE probe's 
internal DAS to be powered down until testing. A transistor-transistor logic (TTL) event marker was utilized to sync the small probe DAS with the AHF data system. When initiated, the small probe DAS entered an interrupt routine to mark the event, which then could be aligned in post data processing.

\section{B. SPRITE Test 1 DAS Performance}

The first SPRITE probe test successfully demonstrated the ability to condition and telemeter TPS sensor data. However three hardware flaws were discovered in post data processing. The first was a TC low pass filter design error. Multiple DAS filters were misaligned introducing electrical noise to the measurements. This misalignment resulted in an error of up to 20 degrees Celsius at any given time. This sporadic behavior can be seen on TC 24 and TC 27 in Figure 6. This design flaw was identified and repaired for the second probe test.

The second flaw caused the saturation of TC 22 that can be seen in Figure 6. The initial DAS design was specified to measure a type K TC range of approximately -100 to 1200 degrees Celsius. This range was surpassed by TC 22 in test 1 . To rectify this limitation, the gain of the instrumentation amplifier was reduced resulting in a range of approximately -150 to 1400 degrees Celsius.

The third hardware malfunction discovered during post data processing was frequent power cycling of the DAS. During post test analysis it was discovered that the $5 \mathrm{~V}$ regulator was near its max operating temperature in a table top setting due to the power draw of the SPRITE DAS. It was then inferred that in the AHF test chamber under vacuum, the limited convective cooling permitted the regulator to surpass its operating temperature, triggering a thermal shut down until it had returned to a safe operating temperature causing the system to power cycle. This design flaw was confirmed through testing in a vacuum chamber. To fix the problem, a higher performance voltage regulator with an integrated heat sink was employed for the second probe test.

Unfortunately the frequent power cycling did not allow the memory board to reach its 500 byte buffer limit, therefore the data was never written to the memory. However the majority of the data was telemetered and received through the RS-232 connection, providing the SPRITE probe data needed for characterization.

The SPRITE probe test 1 results and analysis can be found in references [2],[3], and [4].
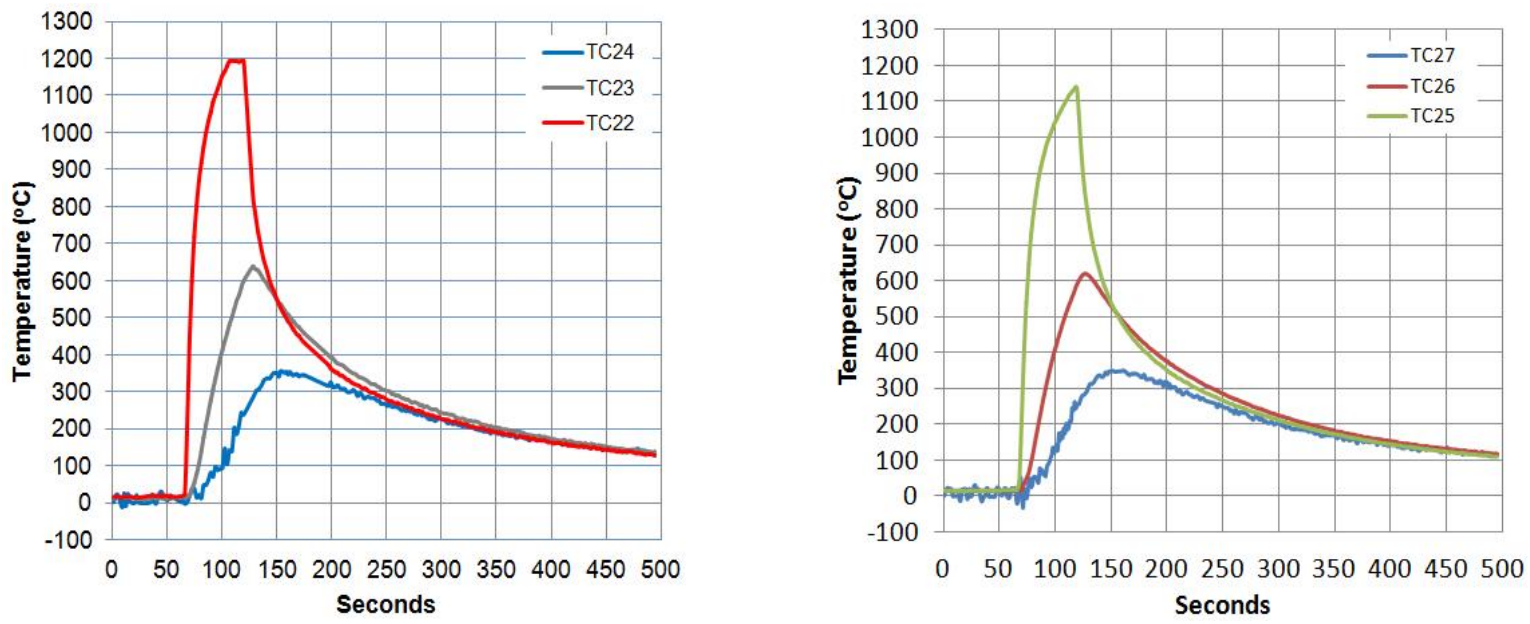

Figure 6. SPRITE probe bottom-cone (right) and mid-cone (left) plug TC plots for test 1.

\section{SPRITE Test 2 Setup and DAS Performance}

The second SPRITE probe was tested at NASA ARC's AHF arc jet in February 2011. This test setup was identical to test 1 with a couple notable exceptions. Although splicing TC signals is not usually practiced, TC 21 was spliced between the SPRITE DAS and the AHF data system to analyze the accuracy of the SPRITE DAS compared to the well calibrated AHF data system. In addition the hardware modifications addressed in the previous section were implemented.

The second probe test successfully demonstrated the SPRITE DAS ability to condition TPS sensors, organize their data, simultaneously write it to on-board memory, and telemeter to a nearby data system. This was accomplished solely using battery power making the SPRITE DAS a stand-alone system. The hardware issues discovered in test 1 were functionally rectified in test 2. As can be seen in Figure 7, TC 24 and TC 27's signal-tonoise ratio had been greatly increased with the filtering correction. Additionally all power cycling issues had been 
eliminated resulting in a fully populated on-board memory file containing all the SPRITE probe sensor data. The test 2 TC data and isotherm data can be seen in Figure 7 and Figure 8.
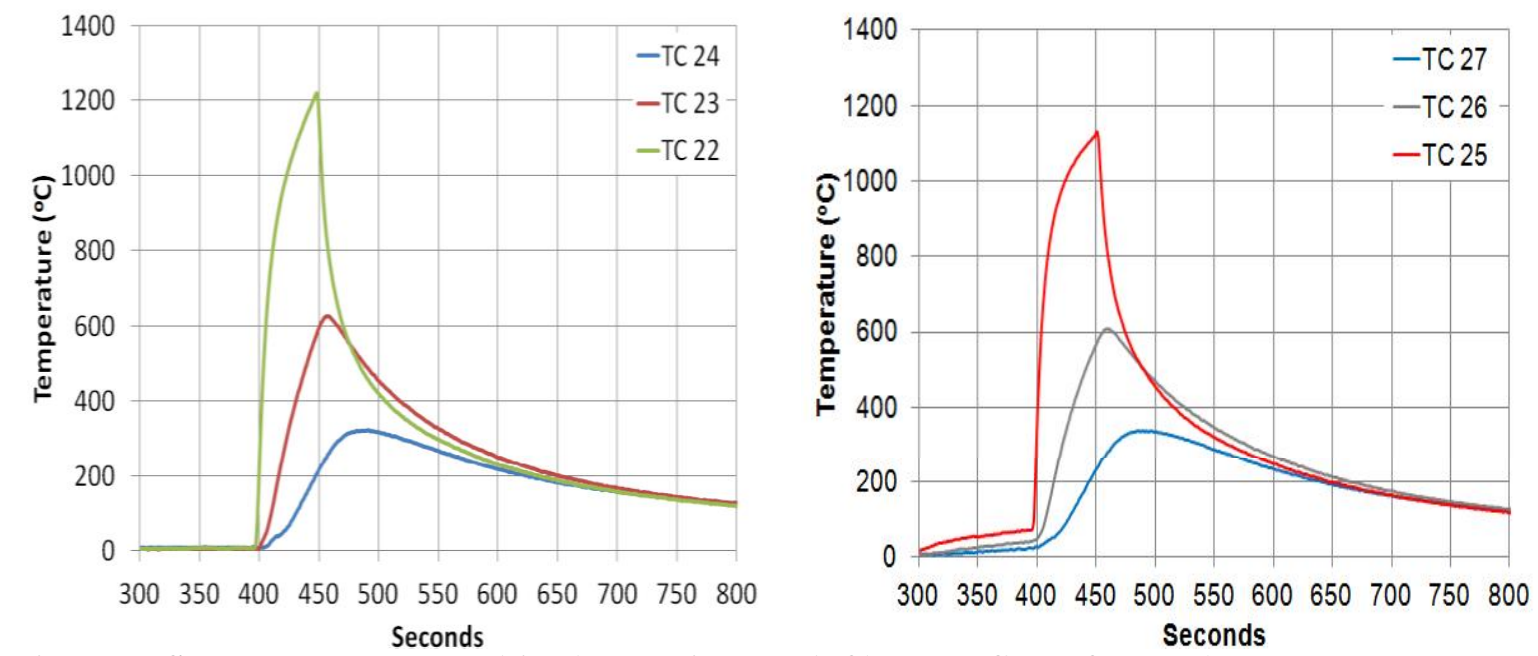

Figure 7. SPRITE bottom-cone (right) and mid-cone (left) plug TC plot for test 2.

Also contained in Figure 8 is the spliced TC measurement accuracy comparison of the SPRITE DAS (TC 21) to the well calibrated AHF data system (TC 14). The SPRITE DAS performed notably with little error, mostly due to the bit resolution of the PIC microprocessor.

The SPRITE probe test 2 results and analysis can be found in references [2], [3], and [4].
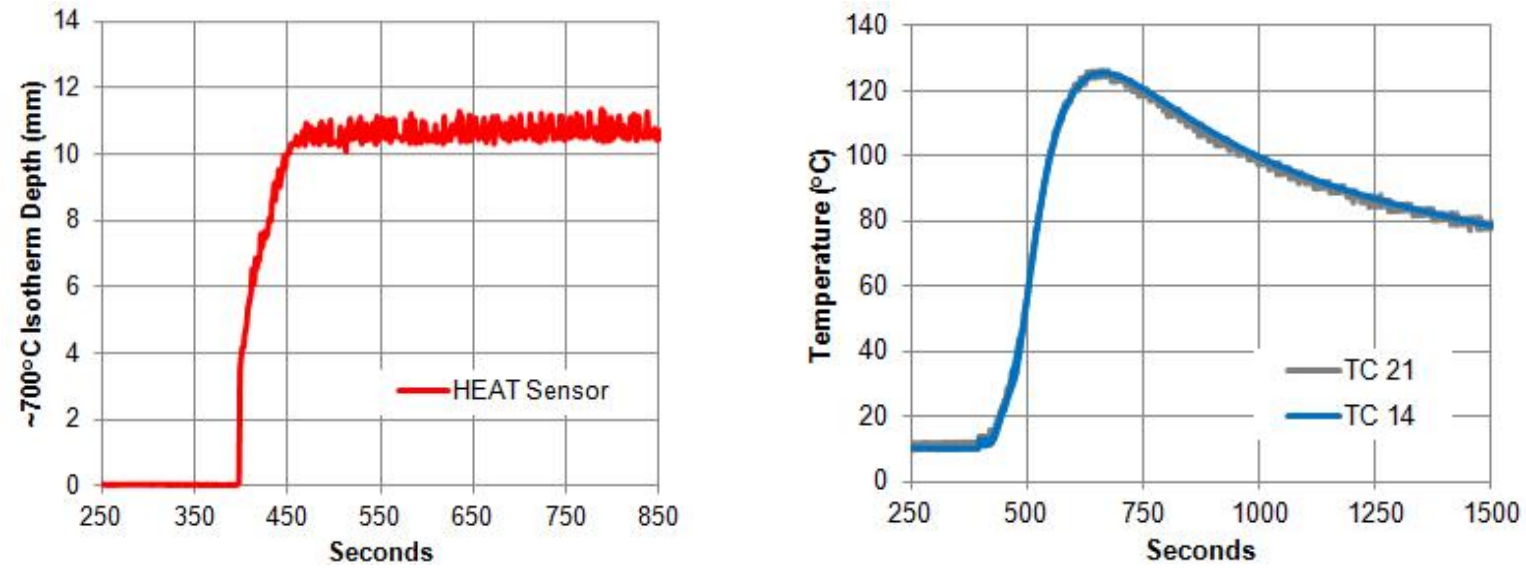

Figure 8. PICA $7^{\circ} 0^{\circ} \mathrm{C}$ isotherm depth measured by the isotherm tracking sensor (left) and the spliced TC measurement comparison between the SPRITE DAS (TC 21) and the AHF data system (TC 14).

\section{Concluding Remarks}

After working through initial hardware design flaws discovered in the in the December 2010 test, the SPRITE DAS successfully met all primary requirements. The battery powered SPRITE DAS successfully conditioned, recorded, and telemetered both thermocouple and isotherm sensor data. Future work will address minor issues that resulted in inefficient data organization within the microprocessor software. In addition a more powerful microprocessor will be utilized to increase the computing power of the SPRITE DAS. The DAS will then be redesigned to encompass a modular approach to incorporate a plug-and-play sensor interface for ultimate flexibility. Finally, to prepare for potential flight testing, the DAS components will be optimized to endure the harsh vibration and radiation environments that will be seen during launch, flight, and re-entry of the SPRITE probe. 


\section{Acknowledgments}

A special thanks to the following people for their well appreciated support during the design and fabrication of the SPRITE probe DAS: Alan Cassell, Austin Howard, Dinesh Prabhu, Keith Peterson, Parul Agrawal, Johnny Fu, Jose Santos, Jerry Ridge, Imelda Terrazas-Salinas

\section{References}

1. Howard, Austin R., Prabhu, Dinesh, K., Venkatapathy, Ethiraj, and Arnold, James, O.: "Small Probes as Flight Test Beds for Thermal Protection Materials" Proceedings of the $7^{\text {th }}$ International Planetary Probe Workshop, Barcelona, Spain, 2010.

2. Empey, D. M., Skokova, K. S., Agrawal, P., Swanson, G. T., Prabhu, D. K., Peterson, K. H., Winter, M., and Venkatapathy, E.. "Small Probe Reentry Investigation for TPS Engineering (SPRITE)," 50th AIAA Aerospace Sciences Meeting, Nashville, TN, January 2012.

3. Skokova, K. S., "Arc Jet Testing of a Small Entry Probe (SPRITE)," 50th AIAA Aerospace Sciences Meeting, Nashville, TN, January 2012.

4. Agrawal, P., Chen, Y.-K., and Prabhu, D.K., "Thermal soak analysis of SPRITE probe," 50th AIAA Aerospace Sciences Meeting, Nashville, TN, January 2012.

5. Venkatapathy, E., Prabhu, D., Skokova, K, Peterson, K., Agrawal, P., Swanson, G., and Empey, D., "Overview of Recent Advancements in Small Probe Reentry Investigation for TPS Engineering (SPRITE)," 50th AIAA Aerospace Sciences Meeting, Nashville, TN, January 2012.

6. Oishi, Tomo, Martinez, Edward, R. and Santos, Jose, A. "Development and Application of a TPS Ablation Sensor for Flight", 46th AIAA Aerospace Sciences Meeting and Exhibit Paper AIAA 2008-1219, Reno, NV, January 2008. 